\title{
Corporeidad, motricidad y propuestas pedagógico-prácticas en aulas de educación infantil
}

\author{
Rodrigo Alberto Gamboa-Jiménez, Ph.D. \\ Pontificia Universidad Católica de Valparaíso, Chile * \\ Milenka Noemí Bernal-Leiva, Lic. \\ Universidad Andrés Bello, Chile** \\ Macarena Paz Gómez-Garay, Lic.

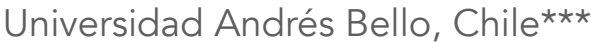

\author{
María José Gutiérrez-Isla, Lic.

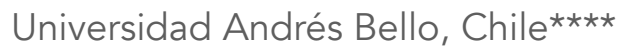 \\ Catalina Belén Monreal-Cortés, Lic. \\ Universidad Andrés Bello, Chile ${ }^{\star \star \star \star \star}$ \\ Valentina Verónica Muñoz-Guzmán, Lic. \\ Universidad Andrés Bello, Chile ${ }^{\star \star \star \star \star \star}$
}

\section{Resumen (analítico)}

Este estudio cualitativo tiene por objetivo analizar la relación que se presenta entre el discurso y las propuestas pedagógico-prácticas en torno a la corporeidad y su expresión motriz en aulas de educación infantil. Para la recolección de la información se aplicaron entrevistas y observaciones a un grupo de educadoras de párvulos que se desempeñaba en tres realidades educativas de la ciudad de Viña del Mar, Chile. El análisis de la información se realizó desde una lógica inductiva interpretativa, siguiendo las directrices de fragmentación y articulación de la teoría fundamentada. Los hallazgos revelan tensiones entre discursos que valoran la corporeidad y su expresión motriz en edades tempranas, y lo que acontece en la realidad del aula infantil: prácticas corporales basadas en una visión tradicional, tecnocrática y funcional de la Educación Física.

\section{Palabras clave}

Educación de la primera infancia, Infancia, prácticas pedagógicas, educación física.

\section{Thesauro}

Tesauro de Ciencias Sociales de la Unesco.

\section{Para citar este artículo}

Gamboa-Jiménez, R. A., Bernal-Leiva, M. N., Gómez-Garay, M. P., Gutiérrez-Isla, M. J., MonrealCortés, C. B., \& Muñoz-Guzmán, V. V. (2020). Corporeidad, motricidad y propuestas pedagógicoprácticas en aulas de educación infantil. Revista Latinoamericana de Ciencias Sociales, Niñez y Juventud, 18(1), 1-22. http://dx.doi.org/ $10.11600 / 1692715 x .18101$

\section{Historial}

Recibido: 22.10.2018

Aceptado: 05.02.2019

Publicado: 04.11.2019

\section{Información artículo}

Este artículo de investigación científica, hace parte del Proyecto de Investigación N 11140872, «Percepciones en torno a la motricidad, la corporalidad y las prácticas corporales en la infancia. ¿Dónde estamos?», financiado por el Fondo Nacional de Desarrollo Científica y Tecnológica, Fondecyt Chile. Estudio cualitativo, descriptivo-interpretativo. Noviembre de 2014-Noviembre de 2017. Área: Ciencias sociales; subárea: Ciencias de la educación. 


\section{Corporality and motricity in early childhood classrooms: the relationship between discourse and praxis}

Abstract (analytical)

This qualitative study analyzes the relationship between discourse and practical pedagogical proposals that cover corporality and its motor expression in early childhood classrooms. For the information gathering process, interviews and field observations were conducted with a group of Early Childhood Educators who work in three educational contexts in the city of Viña del Mar, Chile. The information was analyzed using an interpretative inductive logic following fragmentation articulation guidelines of Grounded Theory. The findings reveal tensions between discourses that value corporality and its motor expression in early ages, and what is happening in the realities of children's classrooms, which are corporal practices based on a traditional, technocratic and functional vision of Physical Education.

Keywords

Early childhood education, childhood, pedagogical practices, physical education.

\section{Corporeidade e motricidade na sala de aula da educação infantil: a relação entre discurso e praxis}

Resumo (analítico)

Este estudo qualitativo visa analisar a relação entre discurso e propostas pedagógico práticas em torno da corporeidade e sua expressão motora nas salas de aula da educação infantil. Para o processo de coleta de informações, entrevistas e observações de campo foram aplicadas a um grupo de educadores da primeira infância que trabalham em três realidades educacionais na cidade de Viña del Mar, no Chile. A análise da informação é realizada a partir de uma lógica indutiva interpretativa, seguindo as diretrizes de articulação de fragmentação de The Grounded Theory. Os achados revelam tensões entre discursos que valorizam a corporalidade e sua expressão motora nas primeiras idades, e o que está acontecendo na realidade da sala de aula das crianças: práticas corporais baseadas em uma visão tradicional, tecnocrática e funcional da Educação Física.

Palavras-chave

Educação infantil, infância, práticas pedagógicas, educação física.

\section{Información autores}

[*] Investigador responsable. Profesor Educación Física. Doctor en Educación por la Universidad de Granada, España. Docente investigador Pontificia Universidad Católica de Valparaíso Chile. iD 0000-0001-6060-5317 Índice H5: 1. Correo electrónico: rodrigo.gamboa@pucv.cl

[**] Licenciada en Educación. Educadora de Párvulos Universidad Andrés Bello Chile. (iD) 0000-0003-4326-5810 Índice H5: 0. Correo electrónico: milenkabernal93@gmail.com

[**] Licenciada en Educación. Educadora de Párvulos Universidad Andrés Bello Chile. (iD 0000-0003-3049-3689 Índice H5: 0. Correo electrónico: macagomez92@gmail.com

[****] Licenciada en Educación. Educadora de Párvulos Universidad Andrés Bello Chile. (iD 0000-0003-4571-3779 Índice H5: 0. Correo electrónico: mariajoseeg.94@gmail.com

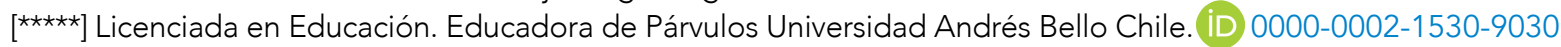
Índice H5: 0. Correo electrónico: catalina_bmc@hotmail.com

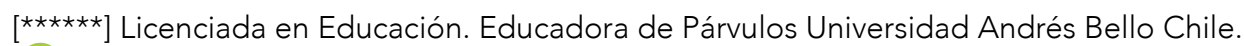

iD 0000-0001-5560-853X. Índice H5: 0. Correo electrónico: valentina-92@hotmail.es 


\section{Introducción}

En los últimos años, la educación física, desde su perspectiva de práctica social y pedagógica, como parte constitutiva del currículo escolar y a la cual se le ha delegado la responsabilidad del ámbito corporal, ha sido emparentada esencialmente con un paradigma científico positivista, donde prevalece una visión tecnocrática, funcional e instrumental del área (Pedraz, 2012); lo anterior con una mirada reduccionista, fragmentada y dualista de la persona, quitándole el valor y sentido a la disciplina (Gamboa-Jiménez et al., 2018; Toro, 2007). Desde esta mirada, el cuerpo es concebido como un instrumento, una máquina (Moreno, 2011), el cual debe ser entrenado sin importar las consecuencias en el bienestar de las personas. Para Giraldes (2013) estas prácticas giran esencialmente en torno a perspectivas biofisiológicas, alejándose del sentido pedagógico de conocer y valorarse, de relacionarse consigo mismo, con su entorno y los demás, base para una vida en relación.

Esta problemática ha sido estudiada por autores como Delgado y Zurita (2002), Moreno y Medina (2012), Poblete y Gamboa-Jiménez (2013), entre otros; investigaciones que indagan sobre las creencias explícitas o implícitas de profesores y estudiantes sobre la educación física en diversos contextos; creencias que concuerdan plenamente con las visiones anteriormente señaladas. Para López et al. (2005) esta visión ha sido reforzada por los mismos profesionales del área y, al parecer, se ha construido por los insuficientes espacios de discusión y puestas en común sobre el real sentido de la disciplina.

En nuestra realidad (Chile) un ejemplo de esta visión dominante es la implementación desde el 2010 del Simce en educación física (Sistema de Medición de la Calidad de la Educación), que evalúa la calidad de las clases a partir de pruebas estandarizadas centradas en la condición física de escolares (Moreno, 2014; Moreno, et al., 2014; Rodríguez, et al., 2015). Esta mirada reduccionista de su rol es corroborada por la Agencia de Calidad de la Educación Chilena (2015), la cual propone, a partir de los resultados del Simce, 
que las clases se trabajen de manera diferenciada según nivel de condición física de los estudiantes.

Estas características también son observadas en las prácticas corporales infantiles, con una mirada reducida, limitada y fragmentada de cuerpo; lógica que conduce a transformar la presencia e interacción de niños y niñas en una relación homogénea, donde lo propio de cada ser se invisibiliza (Gamboa-Jiménez et al., 2018). Mirada que, con preocupación, observamos ha permeado la educación infantil chilena; en ella hemos sido testigos, por ejemplo, de la instalación de minimáquinas de ejercicios al interior de instituciones educativas (República de Arica, 2012) y la importación del proyecto Prefit que se plantea «la necesidad de evaluar con precisión la condición física en niños en edad preescolar, esto es, de 3 a 5 años» (Profit, s.d., §. 1), al cual se lo ha denominado Prefit-Chile.

A nuestro entender las prácticas corporales bajo esta visión imperante en Chile, caracterizada por una estructura rígida, lineal, tradicional y autoritaria, donde se trabaja desde la dualidad cuerpo-mente propias del paradigma cartesiano, se transforman en un espacio de entrenamiento (Almonacid, 2012). Estas prácticas no dan respuesta a la propuesta del nivel de educación infantil, dado que para el Ministerio de Educación [Mineduc], se debe «favorecer aprendizajes de calidad para todas las niñas y niños en una etapa crucial del desarrollo» y añade, «es importante el desarrollo de aspectos claves como: los primeros vínculos afectivos, la confianza básica, la identidad, la autoestima, la formación valórica, el lenguaje, la inteligencia emocional, la sensomotricidad y las habilidades del pensamiento» (2005, p. 15).

Ahora bien, en los últimos años se pueden observar propuestas que evolucionan hacia una pedagogía centrada en el estudiante, en su autotransformación y la transformación de su contexto (Pascual, 2004; Rivera et al., 2012). Estas han privilegiado un enfoque fundado en la acción y una relación pragmática con el saber (Perrenoud, 2006), tensionando así la visión de la educación física ya mencionada. Propuestas que comprenden que niños y niñas en sus primeros años de vida se contactan con el mundo desde su corporeidad y motricidad (Gamboa-Jiménez, et al., 2019; Pikler, 20oo). La corporeidad comprendida como continente de la subjetividad de la persona y la motricidad como expresión de dicha subjetividad, del sello particular de ser y estar en el mundo. Un cuerpo «donde ocurre el acontecimiento del existir» (Gallo, 2009, p. 233) y una motricidad que comprende el 
mover-se como aquel movimiento que deja de ser simplemente un cambio de lugar, por un movimiento que entra en relación con el mundo de la vida, como aquel movimiento que está ligado a la vivencia, las emociones, las percepciones, el estado de ánimo, el sentir corporal, a la conciencia encarnada, es decir, es la motricidad misma. (Gallo, 2006, p. 55)

Desde estas concepciones, al hablar de corporeidad y no de cuerpo, de motricidad y no de movimiento, no lo hacemos por un aspecto decorativo, sino con sentidos y significados. Con ello nos alejamos del paradigma descrito en las primeras líneas de este trabajo y hacemos parte de una mirada integral del ser humano, expresando precisamente la unidad, singularidad y complejidad de nuestra presencia única e irrepetible en el mundo y ante los demás.

Así, consideramos que la infancia es un periodo somatopsíquico, que parte de la acción e interacción con el medio (Aucouturier, 2007), y poseemos la convicción de que la motricidad y corporeidad son la condición misma de existencia, y la expresión de la integración del ser que comienza a florecer. En cuando tales, ambas vividas placenteramente, se encuentran en la base del desarrollo humano y son la puerta de entrada hacia el desarrollo de la autonomía, la identidad, la comunicación, la convivencia y la creatividad (Gamboa-Jiménez et al., 2019); aspectos que se deben constituir en ejes fundamentales y vertebradores de las propuestas educativas del nivel.

De aquí se desprende la importancia de las prácticas corporales en educación infantil, entendidas como una «amplia matriz de manifestaciones motrices intencionadas, realizadas por un sujeto que es uno y complejo, que existe desde una historia personal y colectiva en un contexto socio-cultural presente determinado» (Jiménez, 2015, p. 62); propiciando la creación de ambientes y oportunidades para responder al desafío de una pedagógica centrada en la capacidad de ser, en una dinámica de experimentación y descubrimiento, que consolide desde temprano el conocimiento y valoración de sentirse capaz, hecho vital que constituirá una de las bases del desarrollo. Este es el sentido pedagógico al cual adherimos. Deben ser un espacio para «establecer relaciones de confianza, afecto, colaboración, comprensión y pertenencia, basadas en el respeto a las personas y en las normas y valores de la sociedad a la que pertenece» (Mineduc, 2005, p. 51), así como también una práctica situada, una educación más humana (Maturana, 2006); lo que, en palabras de Osorio (2017), se debe educar:

Desde una ecología del cuidado y de la libertad de ser, crear y convivir desde la convicción de que es posible transformar la educación en un acto emancipador y las escuelas en ex- 
presiones creativas en la red de aprendizajes que constituyen a los seres humanos de todas las edades y condición social y cultural en sujetos de sus propios proyectos de vida. (p. 9)

Para nosotros estas ideas se transforman en necesarias para comprender el desafío profesional de constituirse en mediadores de la educación de los niños y niñas en la infancia (Moreno, 2018), donde la práctica corporal es una de sus variables fundamentales. Esto es, vivencias que les permitan experimentar, conocer y expresar su forma particular de ser y estar en el mundo en beneficio de su identidad (Mineduc, 2005); interactuar positivamente con los demás, configurando su dimensión socioafectiva (Chokler, 2005); y tener la posibilidad de crear, de exteriorizar, toda la energía de innovación en pos del desarrollo del pensamiento divergente (Trigo, 1999).

Al respecto, Vaca y Varela (2008), desde un análisis global de la jornada de trabajo y las presencias del cuerpo en el aula de educación Infantil, ponen en tensión estas últimas ideas expuestas en cuanto la vivencia corporal como posibilidades de desarrollo y el rol de los educadores al respecto. Concluyen que, desde los cuerpos silenciados en las experiencias de aprendizaje, hasta los cuerpos sueltos en los espacios de recreos, los primeros son los que predominan en las escuelas.

En este marco de ideas, se plantea como objetivo el indagar en las percepciones que poseen educadoras en torno a la corporeidad y la motricidad; así como analizar la relación que se establece con sus propuestas pedagógico-prácticas vinculadas a dichas temáticas. Ello con el propósito de la elaboración argumentada de información relacionada con la dialéctica que se da entre la teoría, los discursos y dichas propuestas pedagógicas de lo corporal en las aulas de educación infantil; entendidas estas como las propuestas de tareas de aprendizaje realizadas por las educadoras a niños y niñas.

\section{Método}

De acuerdo al objetivo señalado y pregunta que nos orienta, ¿cuáles son las relaciones que se establecen entre la teoría, los discursos y las propuestas pedagógico-prácticas de educadoras en el ámbito de lo corporal en aulas de educación infantil?, la presente investigación es cualitativa. Posee un carácter descriptivo-interpretativo y diseño fenomenológico, dado que busca comprender los significados que los sujetos le otorgan al fenómeno estudiado desde la experiencia vivida, relevando su valor como personas (Jaramillo \& Dávila, 2012). 
Los sujetos participantes fueron educadoras de párvulos (mujeres) que desempeñaban un rol pedagógico en jardines infantiles de la ciudad de Viña del Mar Chile, pertenecientes a la Junta Nacional de Jardines Infantiles [Junji] (1) y particulares (2). Todas con títulos profesionales en universidades tradicionales pertenecientes al Consejo de Rectores de las Universidades Chilenas (Cruch), sin estudios de posgrado. Las dos instituciones de dependencia administrativa particular atienden a niños y niñas de sectores socioeconómicos de clase media, mientras que la de dependencia pública a niños y niñas de clase socioeconómica media-baja.

\section{Tabla 1}

Participantes $^{1}$

\begin{tabular}{ccc}
\hline Nombre & Dependencia & Años de experiencia \\
\hline Alpina & Particular & 15 \\
Onix & Particular & 25 \\
Adara & JUNJI & 20 \\
\hline
\end{tabular}

Dichas instituciones fueron seleccionadas a partir de los siguientes criterios: i) jardines infantiles que colaboran en el proyecto Fondecyt, en el que se enmarca el presente trabajo; ii) disposición y factibilidad de participar por parte de las instituciones; iii) consentimiento de las familias; y iv) accesibilidad para los investigadores.

Conjuntamente a la revisión bibliográfica que otorgó sustento teórico al estudio, y en coherencia a la opción metodológica seleccionada, se realizaron entrevistas en profundidad semiestructuradas; estas, gracias a su carácter comunicativo cara a cara (Flick, 2004), nos permitieron adentrarnos en las percepciones de los sujetos en torno a la temática de estudio (Araneda, 2008). Las entrevistas se basaron en un guión, lo que posibilitó organizar, eliminar e incorporar preguntas según cómo se desarrolló el proceso (Bernal, 2006).

También se realizaron observaciones de los espacios pedagógicos destinados al ámbito de lo corporal de las educadoras parte del estudio. Se observaron las tres realidades por un periodo de 5 meses, una vez a la semana, por una hora (tiempo que duraban las sesiones de trabajo). Ello nos permitió obtener un conjunto de registros sistemáticos de patrones de conducta, que se constituyeron en información relevante del fenómeno estudiado (Pedret, et al., 2000). La observación fue de tipo participante pasiva, debido a

\footnotetext{
${ }^{1}$ Nombres ficticios.
} 
que los observadores estuvieron presentes sin intervenir directamente en el contexto (Flick, 2004).

Las notas de campo permitieron describir y analizar las presencias corporales de los niños y niñas en el aula; esto, a partir de las propuestas pedagógico-prácticas de las educadoras entrevistadas. La saturación de la información fue un indicador clave para finalizar este proceso; es decir, no se presenciaron nuevos acontecimientos que permitieran obtener nuevas y diferentes conclusiones.

El análisis de la información, que implicó un conjunto de exploraciones, transformaciones y reflexiones sobre la información recolectada, con el propósito de obtener significados relevantes del problema estudiado (Rodríguez et al., 1999), se realizó a través de un proceso de categorización. A las categorías se las entendió como tendencias en las que se clasifica la información (Latorre, 2007). Se siguieron las directrices de fragmentación y articulación de la teoría fundamentada (Strauss \& Corbin, 2002), desde una lógica inductiva-interpretativa y categorías emergentes.

Las entrevistas y observaciones fueron transcritas a un formato archivo digital Word $^{\mathrm{TM}}$ e importadas el software Nvivo $11^{\mathrm{TM}}$; en este último nos apoyamos para el proceso de análisis que constó de tres etapas de codificación:

i) Abierta: permite clasificar y reducir la información e identificarla a través de temáticas. Se constituye en un "proceso analítico por medio del cual se identifican los conceptos y se descubren en los datos sus propiedades y dimensiones» (Strauss \& Corbin, 2002, p. 110).

ii) Axial: permite sintetizar la información considerando las recurrencias, agrupando las temáticas bajo un mismo tópico. Implica la transformación de los datos (Rodríguez et al., 1999).

iii) Selectiva: permite reagrupar los tópicos de acuerdo con recurrencias, bajo una misma categoría; identificando los hallazgos más relevantes del estudio. Para las observaciones, esta etapa realizó según las presencias corporales en el aula propuestas por Vaca y Varela (2008).

Finalizadas las tres etapas construyó un modelo comprensivo-interpretativo, que permitió constatar de manera gráfica los distintos hallazgos.

Para resguardar los principios y valores de toda investigación, podemos señalar como principales criterios éticos (Rodríguez et al., 1999): 
i) Confidencialidad de información e informantes.

ii) Conocimiento del informe por parte de los sujetos.

iv) Derecho a solicitar retiro parcial o total de la información por disconformidad de esta por parte de los sujetos.

v) Firma de consentimiento informado.

\section{Resultados}

De la etapa de codificación abierta, se obtuvieron una gran cantidad de temáticas, que, por razones de espacio, no se mencionan.

A partir de la segunda etapa de codificación obtuvimos los tópicos y porcentaje de referencia obtenidos de las entrevistas y observaciones. Los factores que inciden en los procesos educativos y las posibilidades de exploración sensoriomotriz son los tópicos con mayor presencia en los discursos de las educadoras (28.89\% y $22.22 \%$ respectivamente). Las áreas de desarrollo posibles son temáticas que también ocupan un lugar importante (18.89\%). Con menos presencia se encuentran el rol de los participantes del proceso y las posibilidades de prácticas pedagógicas corporales (10 \% y $13.33 \%$ ). Finalmente, las entrevistadas dedican solo un $6.67 \%$ para referirse a los posibles aprendizajes a lograr.

Del proceso de las observaciones las tareas de exploración, de habilidades motrices básicas y tareas relacionadas con el cuerpo, poseen la mayor presencia en las propuestas prácticas de las educadoras ( $21.61 \% ; 18.92 \%$ y18.91\% respectivamente).

Por su parte, las tareas de compartir (5.41\%), representación (8.11\%), higiene (5.41\%), dibujo $(5.41 \%)$, danza $(5.41 \%)$ y conciencia corporal $(8.11 \%)$, poseen presencias semejantes, pero menores a las anteriormente señaladas. De manera marginal, aparecen tareas relacionada con figuras geométricas $(2.7 \%)$.

Para la tercera etapa de codificación se observó que la categoría procesos educativos de la motricidad es la que posee mayor presencia (38.55\%). Aprendizajes y áreas de desarrollo y factores que inciden en los procesos educativos, se sitúan con un alto porcentaje (27.71\% y $22.89 \%$ respectivamente), y con un $10.85 \%$, el rol que asumen los diferentes participantes del proceso educativo.

Por otro lado, en relación a las presencias corporales observadas en las aulas de las educadoras entrevistadas a partir de sus propuestas pedagógico-prácticas (denominación a partir de la propuesta de Vaca y Varela, 2008), es interesante observar que el cuerpo 
objeto de tratamiento educativo, aquel presente en propuestas relacionadas con temáticas propias del área, está presente con un $42.67 \%$.

Cuerpos implicados (aquel que, a partir de las propuestas pedagógicas no establece restricción o negación a la motricidad) y cuerpos silenciados (aquel que se inmoviliza y su expresión motriz es disminuida al mínimo) comparten presencias similares: $29.33 \%$ y $20 \%$ respectivamente.

Respecto a los cuerpos objeto de atención, aquel presente en tareas relacionadas con alimentación y cuidado personal, y cuerpos instrumentados, que posibilita explicar conceptos y construir aprendizajes de otras áreas, constituyen presencias marginales (5.33 \% y $2.67 \%$ ).

A partir del análisis hasta aquí realizado, en que se ha trabajado bajo una lógica de espirales de profundización (que permite avanzar y retroceder de modo de enriquecer el proceso investigativo), se construye un modelo interpretativo (figura 1), que nos ha permitido constatar de manera gráfica los distintos hallazgos. Dicho modelo se construye ubicando en el centro «Corporeidad y motricidad en el aula de educación infantil», que se constituye como idea central del análisis; y de ahí se desprende en orden jerárquico hacia los extremos, en tres niveles: categorías, tópicos y temáticas.

Figura 1

Modelo comprensivo-interpretativo

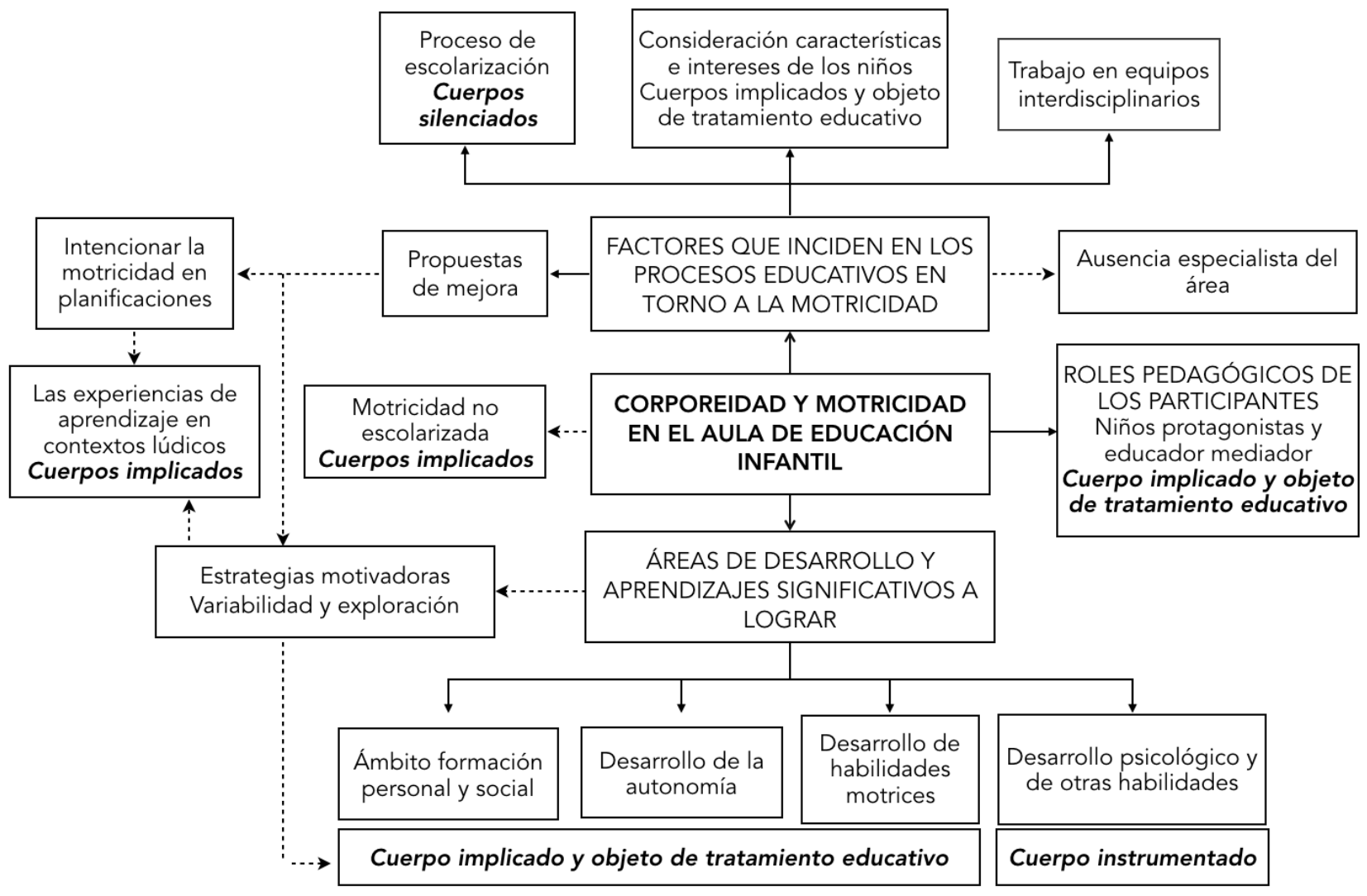




\section{Discusión}

Del modelo anteriormente expuesto, se desprenden tres líneas de interpretación en torno a ideas claves, lo cual permite en este apartado profundizar en los hallazgos más relevantes del estudio, los cuales tensionan la relación dialéctica que se da entre la teoría, los discursos y las propuestas pedagógico-prácticas de las educadoras parte del estudio.

\section{La corporeidad y su expresión motriz se constituyen como fundamentales en el logro de distintos aprendizajes}

Desde las voces de las entrevistadas, la corporeidad y motricidad se visualizan como ejes vertebradores de las propuestas pedagógicas en la infancia; por tanto, las prácticas corporales son esenciales para la construcción de distintos aprendizajes. «Niños y niñas asocian y establecen conceptos de su propio entorno en la medida que lo explora. Siempre he pensado que el aprendizaje se vive con el cuerpo» (Adara, entrevista 3). Sin perjuicio de ello, desde las observaciones los cuerpos instrumentados solo aparecen en dos propuestas educativas $(2.67 \%)$, situación que nos plantea una baja presencia de un trabajo pedagógico globalizador - propio de este nivel-, en el cual el currículo propone no distinguir áreas o ámbitos de desarrollo.

Cada niña y niño es una persona esencialmente indivisible, por lo que enfrenta todo aprendizaje en forma integral, participando con todo su ser en cada experiencia. Construye sus aprendizajes desde sus sentidos, su emoción, su pensamiento, su corporalidad, su espiritualidad, sus experiencias anteriores, sus deseos. (Mineduc, 2018, p. 31)

En esta etapa de la vida —concebida como la fundante del desarrollo humano- los niños y niñas entran en contacto con el mundo, con su propia realidad y con la de quienes lo rodean, desde su corporeidad (Chokler, 2005); por tanto, la percepción del cuerpo y su expresión motriz deben ser valoradas desde la unidad e integridad de la naturaleza humana, como fuentes de expresión y lugar de encuentro con su mundo interior (Montoya \& Trigo, 2015). Presencia, forma de lenguaje, donación y comunicación, que se constituye como valor en el encuentro con los demás (Gallo, 2006; Martin, 200o) y fuente de expresión creativa, innovación y manifestación visible de la fantasía (Gamboa-Jiménez et al., 2019). «No hay nada que podamos decir, sentir, pensar o desear, en lo que no esté implicado nuestro cuerpo» (Gallo, 2009, p. 232). 
Se visualiza a la niña y el niño como una persona en crecimiento, que desarrolla su identidad, que avanza en el descubrimiento de sus emociones y potencialidades en un sentido holístico; que establece vínculos afectivos significativos y expresa sus sentimientos; que desarrolla la capacidad de exploración y comunicación de sus experiencias e ideas, y que se explica el mundo de acuerdo a sus comprensiones, disfrutando plena y lúdicamente de la etapa en que se encuentra. (Mineduc, 2005, p. 15)

En este marco, las prácticas corporales deben ser fundamentadas y realizadas desde una perspectiva unicista del ser humano, donde prime la unidad de la persona como expresión de su esencia natural y cultural (Jiménez, 2015). Por tanto, debiesen alejarse de la visión imperante de la educación física en Chile, donde los objetivos se reducen en aprender a: i) moverse mejor desde la repetición de estereotipos externos; ii) lograr mejores niveles de salud desde una perspectiva biomédica de dicho concepto; y iii) una obsesión por la enseñanza de los deportes (Moreno et al., 2014); elementos presentes en ideas de las educadoras entrevistadas. «Lo importante es que aprendan a ejecutar de manera correcta los movimientos que una les enseña» (Alpina, entrevista 1).

Estamos trabajando aspectos más relacionados con distintas áreas del deporte o donde yo puedo descubrir ciertas habilidades que un niño el día de mañana podría derivarse a que sea un buen futbolista, que sea un buen nadador, a lo mejor un basquetbolista. (Onix, entrevista 2)

Por su parte, Adara plantea: «El tema de cuidarse y hacer deporte, de alimentarte bien [...] lo estamos trabajando más como para ese lado, de la vida saludable» (entrevista 3). Así, circunscribe la vida saludable a hacer deportes y alimentarse bien; desconociendo aspectos del contexto que pueden incidir en los niveles de bienestar de las personas (Gracia, 1991).

Si bien uno de los sentidos de las prácticas corporales en la escuela debe ser el contribuir a la educación de hábitos de vida sana, se debe favorecer también el desarrollo habilidades sociales, emocionales, motrices, entre otras (Gómez et al., 2008), que tengan el propósito de colaborar en el desarrollo integral de las persona considerando toda su complejidad (Devís, 1995). Y, desde allí, «adquirir en forma gradual una autonomía que le permita valerse adecuada e integralmente en su medio, a través del desarrollo de la confianza y de la conciencia y creciente dominio de sus habilidades corporales, socioemocionales e intelectuales» (Mineduc, 2005, p. 39). 
En este contexto, el jugar para las entrevistadas se constituye como fundamental en los procesos de aprendizaje: «El jardín debe ser un espacio donde ellos puedan jugar constantemente, y nosotras tenemos una tremenda responsabilidad al respecto» (Onix, entrevista 2).

Yo creo que el jardín infantil, tiene que ser el lugar para que el niño juegue y que se haga consciente que a través del juego él está construyendo aprendizaje y que es una tarea que tenemos que reevaluar y reasignarle un significado importante. (Alpina, entrevista 1)

Para el Mineduc (2005), el jugar se constituye como uno de los principios pedagógicos de sus bases curriculares del nivel. En él se «enfatiza el carácter lúdico que deben tener principalmente las situaciones de aprendizaje» (p. 17); ya que el jugar «cumple un rol impulsor del desarrollo de las funciones cognitivas superiores, de la afectividad, de la socialización, de la adaptación creativa a la realidad. El juego es, a la vez, expresión de desarrollo y aprendizaje y condición para ello» (Mineduc, 2018, p. 32).

La motricidad y el juego son elementos indisolubles, en los que el niño o niña desde su experiencia corporal puede vivenciar por sí mismo la autoconstrucción de aprendizajes y de su propio ser (Huizinga, 2007). Así, la acción de jugar se constituye en «el placer por aprender libremente, por descubrir, por construir con el otro; el placer de construir una relación de convivencia desde el respeto, del afecto» (Adara, entrevista 2).

Estas ideas las hemos podido observar en aquellas propuestas donde fundamentalmente se hace presente un cuerpo implicado. Sin perjuicio de ello, solo constituyen un $29.33 \%$ del total de propuestas observadas. Las demás se enmarcan esencialmente en un modelo tradicional de enseñanza, en el que se deben repetir relaciones preestablecidas por el adulto; lo que para Calvo (2018) se constituye en un proceso de escolarización. Para dicho autor, la posibilidad de establecer nuevas relaciones a partir de la experiencia vivida se constituye en un proceso educativo. Esta situación escolarizante planteada por Calvo se manifiesta en las propuestas donde prevalece la presencia de un cuerpo objeto de tratamiento educativo ( $42.67 \%$ ), en las que las educadoras han planteado el «qué»y «cómo hacer». Por ejemplo: «Se recuerdan los pasos de baile que les enseñé la semana pasada; los repetiremos para luego enseñarles la coreografía que haremos para el acto» (Alpina, observación 7). Por su parte Adara indica:

Pasaremos saltando una cuerda y una tabla con los dos pies, luego hace una voltereta hacia adelante sobre esta colchoneta. Salten los conos dispuestos en el lugar con un y otro 
pie; pasen por debajo de la cuerda de guatita y caminen por la viga hacia delante. (Adara, observación 4 )

\section{Niños, niñas y educadoras: su rol protagónico en el aula de educación infantil}

Un tema de consenso entre las entrevistadas se relaciona con el rol mediador que deben asumir los educadores y el rol protagónico de niños y niñas en los distintos procesos de aprendizaje. Respecto a lo primero, la labor docente «es ayudar a los niños a enriquecer su conocimiento mediante estrategias pedagógicas que les permitan relacionarse de mejor manera con su entorno, con variadas experiencias que le ayuden a ser un individuo libre y seguro de sí mismo» (Almeida, 2006, p. 25). Para ello, las experiencias deben ser «lo más motivadora[s] posible, ir cambiando, ir a través del juego [...] tomando el interés de los niños» (Alpina, entrevista 1).

Las educadoras entrevistadas plantean el imperativo de asumir un rol activo en el aula, participando junto a los niños y niñas de las diferentes experiencias. «Tenemos que participar igual que ellos, demostrarles con el ejemplo [...]; correr con ellos, saltar con ellos» (Alpina, entrevista 1).

Conocer las características de niños y niñas, sus intereses, necesidades y una mayor presencia de cuerpos implicados en el aula, se establece como un imperativo para constituirse en un educador mediado (Mineduc, 2018).

A lo mejor lo que estás planteando no les interesa; tú tienes que tomar su opinión [...]. Es un rol más de mediadora que como de una sola instrucción, sino que ver si realmente lo que tú le quieres enseñar es de interés para ellos; es pertinente al grupo en el que tú estás. (Onix, entrevista 2)

En cuanto a los niños y niñas, se les deben proporcionar las oportunidades necesarias para que descubran y aprendan activamente, y construyan sus propias nociones de la realidad (Yimari, 2012); otorgar posibilidades de un cuerpo presente, implicado. «Para mí», señala Alpina (entrevista 1), «ellos deben ser los protagonistas de sus aprendizajes, deben asumir un rol protagónico en el aula».

Desde el juego libre, desde el aprender por aprender, desde el jugar por jugar, desde el conocer por conocer, una metodología mucho más liberadora, menos conductista, desde el punto de vista que yo no establezco lo que tiene que hacer, cuándo hacerlo, cuándo 
tiene que aprenderlo y que, si no lo hace como yo lo quiero, no es válido [...]. Mirar al niño como un sujeto activo capaz de decir lo que quiere hacer y lo que no quiere hacer, un sujeto con derecho. (Adara, entrevista 3)

Desde una mirada global de las prácticas educativas, las prácticas corporales deben favorecer la creación de ambientes y oportunidades de una propuesta pedagógica centrada en la capacidad de ser del niño o niña, consolidando su conocimiento y valoración de sentirse capaz.

La niña y el niño deben ser efectivamente protagonistas de sus aprendizajes a través de procesos de apropiación, construcción y comunicación. Ello implica considerar que los niños aprenden actuando, sintiendo y pensando [...] experiencias en un contexto en que se les ofrecen oportunidades de aprendizaje según sus posibilidades. (Mineduc, 2005, p. 17)

Estas ideas se hacen necesarias para entender la relación que se establece entre las prácticas corporales y el desarrollo del niño o niña, de la cual se desprende el desafío profesional de constituirse en mediadores de los aprendizajes; transformando el aula en espacios y tiempos vitales de existencia, otorgando oportunidades para:

i) Explorar en libertad el entorno, vivenciando placenteramente la corporeidad y la capacidad de poder hacer en forma independiente y lúdica; cristalizando sus iniciativas motrices singulares.

ii) Despertar la sensibilidad kinestésica, laberíntica, táctil y visual.

iii) Atender selectivamente e interpretar las tareas propuestas.

iv) Exteriorizar su mundo interior e interactuar positivamente con sus semejantes.

v) Compartir significados desde diferentes formas de lenguaje (Gamboa-Jiménez et al., 2019).

Sin embargo, a nuestro entender, esta posibilidad es difícil de implementar si se continuan proponiendo prácticas pedagógicas tradicionales, fundadas en parámetros biofisiológicos, con el único propósito de responder a resultados ajenos a la realidad de niños y niñas, donde lo importante son los resultados objetivos y medibles como el caso de las pruebas estandarizadas (Moreno \& Medina, 2012); las cuales están alejadas de la realidad de la infancia y de los requerimientos del marco curricular del nivel. 


\section{Barreras que inciden en las prácticas corporales en la educación infantil}

Revelemos dos elementos centrales que emergen desde los discursos de las entrevistadas: Prácticas corporales bajo una mirada funcional, instrumental y tecnocrática

Esta mirada, fundamentada en parámetros biofisiológicos, ha permeado al nivel de educación infantil y las funciones de la educación física descritas por Moreno et al. (2014), emergiendo con fuerza desde los discursos de las entrevistadas.

Hemos analizado en puntos anteriores como Alpina (entrevista 1) plantea la necesidad de que niños y niñas aprendan bien los movimientos que se les enseña; Onix (entrevista 2) centra el foco de sus propuestas en futuros aprendizajes deportivos; y Adara (entrevista 3) relaciona la vida saludable con hacer deporte y alimentarse bien. Conjuntamente a ello, se planteó la prevalencia en las observaciones de cuerpos objeto de tratamiento educativo; con tareas que plantean el «qué»y «cómo hacer».

Esta mirada de las prácticas corporales, a nuestro entender, no dan respuesta a la propuesta de este nivel, donde el desarrollo de la autonomía, la identidad, la convivencia y la comunicación son ejes constituyentes de sus bases curriculares (Mineduc, 2005, 2018).

\section{Procesos de escolarización en educación infantil}

Las entrevistadas señalan los procesos de escolarización como una de las principales barreras para una mayor presencia de prácticas corporales en el aula de educación infantil. Para Escobar (2015) la educación infantil ha sido capturada por propuestas de aprendizaje que no son acordes al nivel madurativo de esta etapa; en consecuencia, se interrumpen ciclos cognitivos y emotivos importantes de niños y niñas. «Tenemos que hacer un mea culpa; siento que con nuestras propuestas como educadoras con la idea de prepararlos bien para el colegio, les estamos quitando tiempo para jugar» (Adara, entrevista 3 ).

Existe una preocupación muy grande en los jardines infantiles en general por preparar a los niños y niñas para la enseñanza básica, lo que está cambiando las tareas propias de los niños de estas edades por tareas de lectura y escritura. (Onix, entrevista 2)

Para Peralta (2014) «nadie ha hecho hincapié en uno de los problemas fundamentales de la calidad de la Educación Parvularia, no solo en Chile, sino que también en Latinoamérica, la «sobre escolarización» (§ 2); y añade que «de ser centros lúdicos, de relación y afectos, de descubrimiento y asombro, están siendo reemplazados por aburridos trabajos de lápiz y papel» $\left(\S_{4}\right)$. 
Siguiendo a Peralta, no es solo una problemática de requerimientos del sistema, sino que también «hay muchos problemas de contexto: si tienes 45 niños de 5 a 6 años en una sala y tienes en Junji una educadora cada 2 o 3 salas; o en Integra, una cada 4 o 5 salas» (2002, p. 27). Situación corroborada por la OCDE (2015) y Mineduc (2015), en el análisis sobre indicadores de la educación en Chile.

Frente a esta preocupación, Onix plantea:

Los niños no están como para estar tanto rato sentados escuchándonos, sino que ellos tienen que aprender jugando y eso tiene que ser en todo ámbito de cosas, no solamente en lo motriz, la parte lógico-matemático, lenguaje, todo tiene que ser a través del juego [...] tienen que venir a jugar, a pasarlo bien. (entrevista 2)

Para Manhey (2017), las familias con sus requerimientos y expectativas movilizan a los educadores hacia la realización de experiencias de aprendizaje descontextualizadas y poco pertinentes para edades tempranas. «La mamá también de repente 'bueno es que me dijo que jugó todo el día', sí jugó, pero aprendió los números, aprendió, no sé, el concepto del tema de solidaridad, aprendió» (Onix, entrevista 2).

En este marco de ideas, llama profundamente la atención que el $20 \%$ de las propuestas pedagógicas observadas en espacios destinados para el área corporal se presentan cuerpos silenciados.

\section{Conclusiones}

Creemos importante centrar este apartado en tres ideas:

Se constata la existencia de tensiones entre lo que acontece en la realidad del aula infantil, los discursos de los sujetos participantes, la teoría y los requerimientos del Mineduc. Si bien desde las entrevistas se observa una valoración de la corporeidad y la motricidad, y se afirma la relevancia de su educación, al parecer se queda en lo discursivo. Las propuestas pedagógicas que son narradas y observadas se emparentan con prácticas tradicionales, funcionales, deportivizadas y bajo un enfoque biomédico de la salud; con lo cual se alejan de lo esencial de esta etapa de la vida y de lo sustantivo propuesto por el nivel.

Se debe transitar desde una lógica fragmentada del sistema educativo hacia ejes vertebradores de la experiencia de aprendizaje infantil, respondiendo a la forma en cómo el 
niño o niña se aproxima al conocimiento contextualizado en lo que ellos están encarnando en su vida cotidiana y de relación. Se nos plantea como desafío realizar propuestas pedagógicas que posibiliten cuerpos implicados y cuerpos instrumentados, que permitan caminar hacia un trabajo globalizador, en el cual los niños y niñas sean los protagonistas de sus aprendizajes.

Creemos importante dar oportunidades para que se haga presente en el aula un cuerpo que esté presente en toda la jornada y no solo en momentos dedicados al ámbito corporal, disminuyendo al mínimo los cuerpos silenciados. Esta situación nos hace proyectar un trabajo pedagógico integral propio de este nivel educacional, en el cual no se divida la jornada en ámbitos de desarrollo. Este último punto se constituye como una proyección del presente trabajo.

Finalmente, queremos platear como limitación o elementos no resueltos el porqué, si bien existe una valoración de la corporeidad y su expresión motriz en los procesos de aprendizaje, las educadoras parte del estudio realizan propuestas educativas ancladas en una mirada tradicional, funcional y deportivizadas de las prácticas corporales. Asimismo, ¿por qué se sitúan desde una lógica fragmentada del sistema educativo, invisibilizando la posibilidad de aprender desde lo corporal? Temas que sin duda también se constituyen como proyecciones del estudio.

\section{Referencias}

Agencia de Calidad de la Educación Chilena. (2015). Entrega de resultados Estudio Nacional Educación Física 2014. Gobierno de Chile.

Almeida, F. (2006). Estrategias pedagógicas que utilizan los docentes para la construcción del presente lógico matemático en niños y niñas [Trabajo de grado no publicado]. Universidad Nacional Experimental Simón Rodríguez.

Almonacid, A. (2012). La educación física como espacio de transformación social y educativa: perspectivas desde los imaginarios sociales y la ciencia de la motricidad humana. Estudios Pedagógicos, 38(1), 177-190. http://dx.doi.org/10.4067/

So718-07052012000400010

Araneda, A. (2008). Investigación cualitativa en educación y pedagogía: texto de apoyo a la formación investigativa de estudiantes de pregrado en la formación, desarrollo y evaluación de Proyectos. Universidad Católica de la Santísima Concepción. 
Aucouturier, B. (2007). Los fantasmas de la acción y la práctica psicomotriz. Graó.

Bernal, C. (2006). Metodología de la investigación: para administración, economía, humanidades y ciencias sociales. Pearson Educación.

Calvo, C. (2018). Educación y escolarización: distinción indispensable para el rescate de la intuición en los procesos de aprendizaje. En M. Mendoza, \& A. Moreno (Eds.), Infancia, juego y corporeidad: una mirada al aprendizaje desde el sur global (pp. 124-140). Ediciones de la Junji.

Chokler, M. (2005). Los organizadores del desarrollo psicomotor: del mecanismo a la psicomotricidad operativa. Ediciones Cinco.

Delgado, M., \& Zurita, F. (2002). Estudio de las teorías implícitas de la educación física en la formación inicial en los maestros en las diferentes especialidades. ¿Qué opinan los futuros maestros? Kronos, 1, 34-44.

Devís, J. (1995). Deporte, educación y sociedad: hacia un deporte escolar diferente. Revista de Educación, 306, 455-472.

Escobar, Y. (2015, 7 de julio). La sobre-escolarización y las consecuencias que trae adelantar etapas en los niños y niñas. Facultad de Ciencias Sociales de la Universidad de Chile. http://www.facso.uchile.cl/noticias/113041/sobre-escolarizacion-y-las-consecuenciasque-trae-adelantar-etapas

Flick, U. (2004). Introducción a la investigación cualitativa. Morata.

Gallo, L. (2006). El ser-corporal-en-el-mundo como punto de partida en la fenomenología de la existencia corpórea. Pensamiento Educativo, 38(1), 46-61.

Gallo, L. (2009). El cuerpo en la educación física da qué pensar: perspectivas hacia una educación corporal. Estudios Pedagógicos, 35(2), 231-242. https://doi.org/10.4067/ So718-07052009000200013

Gamboa-Jiménez, R. A., Jiménez, G., \& Cacciuttolo, C. (2019). Motricidad infantil: bases y lineamientos para re-crear con los niños y las niñas trayectorias de placer. Ediciones Universitarias de Valparaíso.

Gamboa-Jiménez, R. A., Jiménez, G., Peña, N., Gaete, C., \& Aguilera, D. (2018). Prácticas corporales e innovación en educación infantil (o-6 años): análisis crítico desde la mirada de expertos. Revista Brasileira de Ciências do Esporte, 40 (3), 224-232. https:// doi.org/10.1016/j.rbce.2018.03.010

Giraldes, M. (2013). The Fitness Business Channel [video]. YouTube. https:// www.youtube.com/watch?v=HkHbDDv7aKY 
Gómez, A., Díez, L., Fernández, J., Gorrín, A., Pacheco, J., \& Sosa, G. (2008). Nueva propuesta curricular para el área de educación física en la educación primaria. Revista Internacional de Medicina y Ciencias de la Actividad Física y el Deporte, 8(29), 93-108.

Gracia, D. (1991). Modelos actuales de salud: aproximación al concepto de salud. Labor Hospitalaria, 219, 11-14.

Huizinga, J. (2007). Homo Ludens: el juego y la cultura. Fondo de Cultura Económica. Jaramillo, L., \& Dávila, E. (2012). La identidad como categoría relacional en el tiempolugar de recreo. Pedagogía y Saberes, 37, 191-200.

Jiménez, A. (2015). Actividad física y adulto mayor: estudio de las percepciones de los participantes de los programas de los municipios de la región de Valparaíso-Chile [Tesis doctoral no publicada]. Universidad de Granada.

Latorre, A. (2007). La investigación-acción: conocer y cambiar la práctica educativa. Graó. López, V., García, J., García, A., González, M., López, E., Monjas, P., Pérez, D., \& Archilla, M. (2005). ¿Cuándo tenemos poco prestigio? Mirando la viga en el ojo propio. Causas internas y reflexiones sobre el estatus de la educación física. Retos: Nuevas tendencias en Educación Física, Deporte y Recreación, 8, 11-18.

Manhey, M. (2017). Construcción de identidad desde una perspectiva curricular, En M. Mendoza, \& R. Ferrer, (Eds.), Nuevos ambientes de aprendizaje en educación parvularia (pp. 120-127). Ediciones de la Junji.

Martin, K. (2000). Sportodidaktik zum Anfassen. Verlag Karl Hofmann.

Maturana, H. (2006). El sentido de lo humano. Dolmen.

Ministerio de Educación. (2005). Bases curriculares de la educación parvularia. Maval.

Ministerio de Educación. (2015). Análisis de indicadores educativos de Chile y la OCDE en el contexto de la reforma educacional [Serie Evidencias, 31].

Ministerio de Educación. (2018). Bases curriculares de la educación parvularia. Gobierno de Chile.

Montoya, H., \& Trigo, E. (2015). Motricidad humana: aportes a la educación física, recreación $y$ deporte: España-Colombia. Léeme.

Moreno, A. (2011). Percepciones del profesorado universitario en relación a la función de transformación social de la educación física como asignatura del curriculum escolar: el caso de Chile [Tesis doctoral no publicada]. Universidad de Granada.

Moreno, A. (2014). Dime cuánto rindes y yo te diré la calidad de tus clases de educación física. Revista docencii, 52, 64-70.

Moreno, A. (2018). Colonialidad del conocimiento e infancia lúdica: rescatando los saberes infantiles desde el decir/hacer de los niños. En M. Mendoza, \& A. Moreno, 
(Eds.), Infancia, juego y corporeidad: una mirada al aprendizaje desde el sur global (pp. 13-30). Ediciones de la Junji.

Moreno, A., Gamboa-Jiménez, R. A., \& Poblete, C. (2014). La educación física en Chile: análisis crítico de la documentación ministerial. Revista Brasileña Ciências do Esporte, 36(2), 411-427. https://doi.org/10.159o/So101-32892014000200010

Moreno, A., \& Medina, J. (2012). Escuela, educación física y transformación social. Estudios Pedagógicos, 38(1), 7-11. https://doi.org/10.4067/S0718-07052012000400001

Moreno, A., Rivera, E., \& Trigueros, C. (2014). La educación física en chile: un análisis de las creencias del profesorado de la enseñanza primaria y secundaria. Movimento, 20, 81-96. https://doi.org/10.22456/1982-8918.48098

OCDE. (2015). Education at a Glance 2015: OCDE Indicators. OCDE Publishing.

Osorio, J. (2017). Habitar/Des-habitar, re-significar y transformar las escuelas. Nueva Mirada.

Pascual, C. (2004). Formación del profesorado: reflexión-acción y la ética del trabajo bien hecho. Tándem. Didáctica de la Educación Física, 15, 18-25.

Pedraz, M. (2012). El discurso técnico de la educación física o el techo de cristal. Bosquejo de un debate sobre el código disciplinar de la educación física y su precaria legitimidad. Estudios Pedagógicos, 38(1), 89-109. https://doi.org/10.4067/

So718-07052012000400006

Pedret, R., Sagnier, L., \& Camp, F. (200o). La investigación comercial como soporte de marketing. Deusto.

Peralta, M. (2002). ¿Qué se sabe sobre la calidad de la educación parvularia en Chile? En C. Belleï, \& M. Oliva (Eds.), Ciclo de debates: desafíos de la política educacional, ¿qué se sabe sobre la calidad de la educación parvularia chilena? Unicef; Universidad de Concepción.

Peralta, M. (2014, 4 de mayo). La sobre escolarización de la educación parvularia, 2014. Radio Cooperativa. Educación. http://blogs.cooperativa.cl/opinion/educacion/ 20140504080623/la-sobre-escolarizacion-de-la-educacion-parvularia

Perrenoud, P. (2006). Construir competencias desde la escuela. J. C. Sáez. Pikler, E. (200o). Moverse en libertad: desarrollo de la motricidad global. Narcea.

Poblete, C., \& Gamboa-Jiménez, R. A. (2013). Teorías implícitas y proyectos educativos: una visión paradigmática de la educación física. Educere, 17(58), 465-474.

Promoting Fitness \& Health through Physical Activity. (s.d.). Evaluación de la condición física en preescolares. Proyecto Prefit. http://profith.ugr.es/recursos-prefit República de Arica. (2012). Junji implementa máquinas de ejercicio infantil para evitar la obesidad. República de Arica. http://www.republicadearica.cl/?p=121144 
Rivera, E., De la Torre, E., \& Trigueros, C. (2012). (Trans)formar docentes, (trans)formar personas: una experiencia interdisciplinar para democratizar el aula universitaria. Estudios Pedagógicos, 38, 347-37o. https://doi.org/10.4067/So718-07052012000400019 Rodríguez, F., Estrada, D., Quezada, T., Tapia, A., Valderrama, C., \& Moreno, D. (2015). Sistema de medición de la calidad de la educación física en Chile y su influencia en la realidad escolar. Movimento, 21(2), 435-448. https://doi.org/10.22456/1982-8918.49693

Rodríguez, G., Gil, J., \& García, E. (1999). Metodología de la investigación cualitativa. Aljibe.

Strauss, A., \& Corbin, J. (2002). Bases de la investigación cualitativa: técnicas y procedimientos para desarrollar la teoría fundamentada. Universidad de Antioquia.

Toro, S. (2007). Una aproximación epistemológica a la didáctica de la motricidad desde el discurso y práctica docente. Estudios Pedagógicos, 38(1), 29-43. https://doi.org/ 10.4067/So718-07052007000100002

Trigo, E. (1999). Juegos motrices y creatividad. Paidotribo.

Vaca, M., \& Varela, M. (2008) Motricidad y aprendizaje: el tratamiento pedagógico del ámbito corporal. Graó.

Yimari, A. (2012). El aprendizaje según Bruner, Piaget y Vigotsky. Scribd. https:// es.scribd.com/doc/21972411/PIAGET-BRUNER-VIGOTSKY 